\title{
Editorial
}

\section{Anaesthesia and leucocyte locomotion}

"... An anaesthetic is not just a special poison of the nervous system, it anaesthetizes all cells, benumbing all the tissues, and temporarily stopping their irritability."

(Claude Bernard, 1875) ${ }^{1}$

The cellular effects of anaesthetic agents were appreciated by Claude Bernard more than a century ago and the possibility that anaesthesia may alter the course of an infection by modifying the host defenses has been under consideration since the beginning of this century. Recent evidence would support the concept that anaesthesia may suppress both cellular and humoral components of immune response. ${ }^{2-6}$ Among the host defence systems, the migration of granulocytes, macrophages and lymphocytes towards the inflamed or injured tissues is one of the earliest events in the body's defence against infection. Any depression of this defender cell migration is likely to allow the invading organisms to become established and enhance the incidence of infection.

The term chemotaxis was first used in 1884, and the initial observations of leucocyte chemotactic locomotion were first made in the nineteenth century and these pioneer observations of Metchnikoff are still relevant. ${ }^{7}$ Further progress in the field was hampered for many years due to a paucity of adequate techniques and the difficulty of reproducing this phenomenon both in vitro and in vivo. The situation was reversed with the introduction of micropore filter technique in 1962 by Boyden. ${ }^{8}$

In this current issue, Erskine et al. ${ }^{9}$ have reported their investigations of an interesting and clinically relevant question - whether the effects of regional anaesthesia on neutrophil migration are different from those due to general anaesthesia in patients undergoing major orthopaedic surgery. These authors conclude that peripheral blood neutrophils during spinal anaesthesia and surgery migrated farther towards complement-derived attractant than neutrophils obtained from patients undergoing surgery under either halothane or isoflurane anaesthesia. These investigators also reported higher plasma adrenaline levels

Tulane University Medical Center, New Orleans, Louisiana. following surgery under general than spinal anaesthesia. They further postulated that these effects may relate to the surgical stress rather than to the direct effects of anaesthesia since volatile anaesthetics may not have been present in the system at the time of the experimental measurements. This statement, however, is not in total accord with the reported data pertaining to the effects of anaesthesia on chemotaxis and the immune response. ${ }^{2-6}$ Although this study has demonstrated significant differences in the migratory responses of peripheral neutrophils following surgery under regional or general anaesthesia, the mechanism responsible for this altered migration is not clear and no clinical recommendations can be made on the basis of the current investigation. While contradictory and inconclusive reports are not uncommon in the field of chemotaxis, it is important to reflect upon the factors that may influence investigations of leucocyte locomotion in general and the effects of anaesthetic agents on such locomotion in particular. It is also pertinent to consider the clinical implications of anaesthetic-induced depression of leucocyte locomotion.

The migration and accumulation of cells at a given locus may occur by mechanisms other than chemotaxis, e.g., a particular locus may contain adhesive substances that trap cells. Likewise, cells may show preferential adhesion to like cells rather than to unlike cells. Thus, the cells may migrate directionally, not only in response to chemical gradients but also in response to contact guidance and physical properties of the surfaces on which they move. While chemotactic agents may influence the rate and direction of locomotion, the orientation of cells may be controlled by different mechanisms. Therefore, in order to analyse the mode of leucocyte locomotion with clarity, precise definitions and nomenclature for the locomotor reactions of the cells are necessary.

The locomotor reactions of cells to chemical substances in their environment may be defined as follows:

(a) Chemotaxis - "A reaction where the direction of locomotion is determined by gradients of chemotactic substances in their environment." 10

(b) Chemokinesis - A reaction in which the rate of locomotion of cells - or the frequency of their turning - is determined by the substances in their environ- 
ment. Chemokinesis essentially determines the rate of random migration of cells.

The evidence that the behaviour of the cells in vitro is representative of what happens in living tissues in vivo is circumstantial, although this circumstantial evidence has gained considerable credibility. At the present time, most commonly used assays of leucocyte chemokinesis and chemotaxis include migration through micropore filter papers, ${ }^{8}$ migration under agarose, ${ }^{11}$ visual assays, ${ }^{12}$ and migration through three dimensional gels of collagen and fibrin. ${ }^{13}$

To date, most investigators in the field of anaesthesia and leucocyte locomotion have utilized micropore filter assays. During the assay, cell migration across a micropore filter in response to a gradient of chemoattractants is measured. This method is cheap, easy and rapid, and uses simple apparatus. It allows a quantitative measure of the effects of chemical attractants on cell locomotion. Although these assays have been used widely, finding the best way to quantitate them has proved to be an illusive goal. The results may vary considerably depending upon whether one reports the distance migrated by the leading front of the cells, total migrating cells, or number of cells past a given distance from their starting point. Although these measures may provide a valid comparison of cell migration under specific experimental conditions, typical quantities measured in the assay are not useful for comparison between different systems or for correlation with in vivo performance. A major disadvantage of this system is that unless special experiments are set up by using varying concentrations of chemotactic agents both above and below the filters, it does not measure true chemotaxis and one cannot distinguish whether it is the direction of locomotion or the rate of locomotion that is being affected. In their study, Erskine et al. have utilized the raft system described initially by Addison and Babbage. ${ }^{14}$ In this system, the handling of membranes is reduced to a minimum, and since the membranes stay flat, an even layer of cell contact is easily achieved. Also, since several cups containing cells can be placed on the same membrane, a uniformity of experimental conditions may be accomplished. However, migration through membrane systems is not truly representative of the migration of leucocytes through body tissues in vivo.

Additionally, there are other sources of inaccuracies that may influence the experimental data in measurements of chemotaxis in this system as well. Establishment of different chemotactic gradients across the micropore filters, maintaining an optimal pH between 7.2 and 7.4, the type of chemotaxins used, the type of glassware used, fluid levels in the upper and lower chambers, and variations in the different batches of filters, may all contribute towards inaccuracies in measurement of chemotactic migration. In the studies of effects of anaesthesia, additional compounding variables such as the concentrations of the drugs used and their relevance to the clinical concentrations, the effects of these drugs on the $\mathrm{pH}$ of the experimental set up, the presence or absence of serum proteins in the system, the concentration of the free drug etc., need further consideration in the design and interpretation of the experimental data. In the case of volatile anaesthetic agents, it may be virtually impossible to maintain varying concentrations of the drug within one experimental set up, likewise it may be difficult to maintain the same concentration of the drug during the entire procedure. Because of these reasons, while several studies have addressed the issue of anaesthetic effects on leucocyte chemotaxis, experimental data have failed to achieve a uniform consensus and conclusion. Future studies should utilize more recent techniques of neutrophil migration through collagen and fibrin gels that truly simulate migration through human tissues. ${ }^{13}$

A review of the literature reveals that, initially, a reversible dose-dependent inhibition of random locomotion of tetrahymena pyriformis, and peripheral blood human lymphocytes following exposure to halothane was observed by Nunn et al. ${ }^{15}$ By using a modification of Boyden's technique, where directional migration of leucocytes across a micropore filter was measured towards a gradient of a chemo-attractant, Moudgil et al. were first to report a dose-dependent depression of leucocyte migration by local intravenous and volatile anaesthetic agents. ${ }^{16}$ This depression was seen at clinical concentrations of the anaesthetic agents and was short-lived and reversible. However, these authors drew the conclusion that the action of drugs was primarily upon the mechanism of locomotion rather than upon specific cell-detector systems for the chemotaxis. Likewise, an inhibition of leucocyte migration in the postoperative period following surgery under general anaesthesia was initially reported by Stanley et al., ${ }^{17}$ and subsequently confirmed by Moudgil et al. ${ }^{18}$ In contrast, in vitro studies by Duncan and Cullen ${ }^{19}$ and Nunn et al. ${ }^{20}$ failed to reveal a depression of chemotactic migration by thiopentone or $2 \%$ halothane. In these studies, varying concentrations of anaesthetic agents and different methods of measurement were utilized and this may have resulted in a difference in the results of leucocyte locomotion. In order to clarify these contradictory findings, Moudgil et al. investigated the effects of equipotent concentrations of different volatile anaesthetic agents and $70 \%$ nitrous oxide on polymorphonuclear cells and monocyte chemotactic migration. $^{21}$ These investigations revealed that after exposure to one MAC (minimum alveolar concentration) of various anaesthetic agents, the degree of depression of chemotactic migration varied with different anaesthetic agents. With the exception of nitrous oxide, this severity 
of depression paralleled the lipid solubility of the anaesthetic agents. Both the neutrophil and the monocyte chemotactic migration was least affected by isoflurane and most affected by methoxyflurane and $70 \%$ nitrous oxide. In addition to volatile anaesthetics and intravenous induction agents, narcotics including morphine, fentanyl, and meperidine have also been incriminated for causing a depression of leucocyte chemotactic migration.

While several studies, including the current study by Erskine et $a l$, have revealed an anaesthetic-induced depression of chemotactic migration, the clinical relevance of these observations is far from clear. The earlier studies indicate that anaesthesia-induced depression of chemotactic migration is short lived and easily reversible. Altered test values measured in peripheral blood samples need not necessarily mean an overall depression of the host defences. They may vary from altered test values, to reduced reserves, or to frank clinical immunosuppression. Since most immune functions have reserve capacity, a decrease in test value may well be without impact on overall immune competence. While Erskine et al. have demonstrated a difference in chemotactic response between spinal and general anaesthesia groups and have speculated on the mechanism of this difference, the overall impact of such depression on the immunocompetence of a previous healthy population is likely to be negligible. These workers correctly are reluctant to make any clinical recommendations on the basis of their findings. Anaesthesia-induced defects of specific and non-specific components of immune function may, however, be of some relevance in geriatric and paediatric populations, along with traumatized and already immunocompromised patients. Further investigations are necessary in order to define the nature and mechanisms of anaesthesia-induced immune suppression and its ultimate impact on the patient outcome in the perioperative period. Such an understanding may contribute towards a decrease in morbidity and mortality from infection through use of optimal anaesthetic agents in the perioperative period.

\section{L'anesthésie et la locomotion leucocytaire}

«... Un anesthésique est non seulement un poison spécial du système nerveux, il anesthésie toutes les cellules, engourdit tous les tissues et enraye temporairement leur irritabilité. »

\section{(Claude Bernard, 1875) ${ }^{1}$}

Il y a plus d'un siècle, Claude Bernard entrevoit les effets des anesthésiques sur la cellule. Dès 1903, Snell évoque la possibilité que l'anesthésie puisse altérer l'évolution d'une infection en affaiblissant les défenses de son hôte. La littérature récente supporte le concept d'une anesthésie inhibitrice des composantes humorales et cellulaires de la réaction immunitaire. ${ }^{2-6}$ Parmi les défenses immunitaires, la migration des granulocytes, macrophages et lymphocytes vers les tissus lésés par l'inflammation ou l'infection représente la première ligne de défense de l'organisme. Toute défaillance de la défense chimiotactique favorise l'installation d'organismes hostiles et l'augmentation subséquent de l'incidence des infections.

En 1884, Pfeffer utilise pour la première fois le terme chimiotactisme. Les premières observations de la locomotion chimiotactique leucocytaire datent du dix-neuvième siècle, nous parviennent de Leber et de Metchnikov et sont toujours d'actualité. L'inefficacité des méthodes de mesure et l'impossibilité de reproduire le phénomène aussi bien in vitro qu' in vivo retardent tout progrès dans ce domaine pendant plusieurs années. ${ }^{7}$ En 1962 , l'introduction par Boyden $^{8}$ de la technique du filtre à micropores renverse cette inertie.

Dans ce numéro du Journal, l'étude d'Erskine et al. ${ }^{9}$ soulève une question intéressante et fort à propos. Les effets de l'anesthésie régionale sur la migration des neutrophiles differrent ils de ceux de l'anesthésie générale chez des patients soumis à une intervention orthopédique majeure ? Ces auteurs concluent que les neutrophiles qui se trouvent en périphérie du flux sanguin pendant la chirurgie sous rachianesthésie se déplacent sur une plus grande distance en direction d'unattractif dérivé complément que les neutrophiles prélevés sur des patients sous halothane ou isoflurane. Ces chercheurs rapportent de plus un taux plasmatique plus élevé d'adrénaline sous anesthésie générale que sous régionale. Comme ces agents volatils auraient pu être absents de l'organisme au moment des mesures, ils émettent l'opinion que cet effet depend plutôt du stress chirurgical que de l'anesthésie. Ce postulat ne concorde toutefois pas complètement avec les données déjà publiées sur les effets de l'anesthésie sur le chimiotactisme et la réponse immunitaire. ${ }^{2-6}$ Bien que cette étude démontre une disparité significative, après la chirurgie, de la réponse migratoire des neutrophiles périphériques entre l'anesthésie régionale et la générale, le mécanisme responsable de ce changement demeure obscur. On ne peut donc faire de recommandations d'intérêt clinique sur la base de l'étude actuelle. Bien que descomptes rendus vagues ou 
contradictoires se côtoyent dans le domaine du chimiotactisme, il faut réfléchir sur les facteurs qui peuvent influencer la recherche sur la locomotion leucocytaire en général et sur les effets des anesthésiques en particulier. Il devient aussi pertinent de discuter des conséquences cliniques du ralentissement de la locomotion leucocytaire provoqué par l'anesthésie.

La migration et l'amoncellement de cellules sur un locus donné peuvent être causées par des mécanismes autres que le chimiotactisme: par exemple un locus particulier peut contenir des substances adhésives qui emprisonnent les cellules. De la même façon, certaines cellules peuvent montrer une préference pour des cellules semblables qui n'existe pas pour des cellules dissemblables. Ainsi, la migration des cellules peut-être contrôlée non seulement par des gradients chimiques, main peut aussi s'orienter par contact, grâce aux propriétés physiques des surfaces sur lesquelles les cellules se déplacent. Alors que les agents chimiotactiques influenceraient la vitesse et la direction de la locomotion, l'orientation des cellules pourrait être sous le contrôle de mécanismes différentes. Donc pour analyser le mode de locomotion leucocytaire avec intelligence, des définitions précises et une nomenclature des réactions locomotrices deviennent nécessaires.

Les réactions locomotrices des cellules en rapport avec les substances chimiques de leur milieu peuvent se définir comme suit :

a) Chimiotactisme: « une réaction en vertu de laquelle la direction du mouvement est déterminée par des gradients des substances (chimiotactiques) présentes dans le milieu $" .{ }^{10}$

b) Chimiokynèse : une réaction dans laquelle la vitesse de locomotion des cellules - ou la fréquence de leur changement de direction - est déterminée par la nature des substances qui les entourent. Le chimiokynèse détermine le pourcentage de migration des cellules à la dérive.

Bien qu'elle ait gagné beaucoup de crédibilité, l'évidence selon laquelle le comportement des cellules in vitro correspond à leur comportement in vivo est circonstancielle. A l'heure actuelle, les tests de chimiokynèse et de chimiotactisme les plus communéments utilisés comprennent la mesure de la migration des cellules à travers un papier filtre à micropores, ${ }^{8}$ la migration sous agarose, ${ }^{11}$ les épreuves visuelles, ${ }^{12}$ et la migration à travers des gels tridimensionnels de collagène et de fibrine. ${ }^{13}$

Jusqu'à maintenant, la plupart des chercheurs intéressés par l'association anesthésie - locomotion leucocytaire ont utilisé le test des filtres à micropores, qui consiste à mesurer à travers le filtre la réponse de la migration cellulaire à un gradient de substances chimio-attractives. Cette méthode peu coûteuse, facile et rapide n'utilise que du matériel simple. Elle permet une mesure quantitative de l'influence des attractifs chimiques sur la locomotion cellulaire. Bien que ces épreuves soient utilisées un peu partout, il est difficile de trouver un bon moyen de les quantifier. Les résultats varient considérablement selon qu'on rapporte la distance parcourue par la ligne de front des cellules, la totalité des cellules migratrices, ou le nombre de cellules qui ont parcouru une distance déterminée. Bien que ces mesures puissent valider des comparaisons sous des conditions expérimentales spécifiques, les valeurs typiques mesurées par le test ne sont pas comparables avec celles obtenues par d'autres méthodes ou des études in vivo. A moins qu'on ne puisse grâce à des protocoles appropriés qui utilisent des concentrations variées d'agents chimiotaxiques au-dessus ou en-dessous des filtres, la méthodes ne mesure pas la chimiotaxie réelle : on ne peut distinguer alors si c'est la direction de la locomotion ou sa vitesse qui est affectée. Dans leur étude, Erskine et al. ont utilisé la méthode du radeau (raft system) décrite par Addison et Babbage. ${ }^{14}$ Dans celle-ci, la manipulation des membranes est réduite au minimum et comme celles-ci demeurent aplaties, elles restent en contact avec une couche uniforme de cellules. De plus, avec plusieurs contenants de cellules appliquées sur une seule membrane, on peut atteindre une certaine uniformité de conditions expérimentales. Cependant on ne considère pas la migration à travers ces systèmes artificiels comme vraiment représentative de la migration leucocytaire à travers les tissus in vivo.

On trouve aussi d'autres sources d'erreurs susceptibles d'influencer les mesures du chimiotactisme dans ce montage expérimental. La création de gradients chimiotactiques à travers le filtre à micropores, la difficulté de maintenir un $\mathrm{pH}$ idéal de 7,2 à 7,4 , les chimiotaxines utilisées, la verrerie, le niveau liquide des chambres supérieures et inférieures et le manque d'uniformité qualitative entre lots de filtres, sont tous ces facteurs qui contribuent à créer des imprécisions sur les mesures de la migration. Pour les études qui s'appliquent à l'anesthésie, il faut inclure des variables comme la concentration des agents et leur corrélation avec la clinique, les effets du $\mathrm{pH}$, la présence ou l'absence de protéines sériques, la concentration des drogues libres, entre autres, dont il faut tenir compte dans l'élaboration du modèle expérimental et l'interprétation des résultats. Dans le cas des anesthésiques volatils, il peut s'avérer impossible de maintenir une concentration uniforme pendant toute la durée de l'expérience. Pour ces raisons, et malgré le fait que plusieurs études aient examiné les effets de l'anesthésie sur le chimiotactisme leucocytaire, les données expérimentales n'ont pas réussi à créer de consensus et de conclusions valables. Les études futures devraient faire appel aux techniques les plus récentes de migration leucocytaire à travers des gels de fibrin et de collagène qui simulent encore mieux la migration à travers les tissus humains. ${ }^{13}$

Une revue de la littérature révèle que Nunn et al..$^{15}$ ont 
observé une inhibition du mouvement aléatoire du tetrahymena pyriformis et des lymphocytes humains prélevés en périphérie après exposition à l'halothane. Cette inhibition est reversible et liée à la concentration de l'agent. En utilisant une technique de Boyden modifiée où la migration des leucocytes en direction d'un gradient chimioattractif, a été mesurée à travers un filtre à micropores, Moudgil $e t$ al. sont les premiers à rapporter une baisse de la migration leucocytaire proportionnelle à la concentration d'anesthésiques volatils ou la dose d'anesthésique local. ${ }^{16}$ Cette diminution observée à des concentrations cliniques a été brève et reversible. Cependant, ces auteurs en sont venus à la conclusion que l'action des agents se fait sentir sur le mécanisme de locomotion plutôt que sur un système cellulaire de détection propre un chimiotactisme. Plus récemment, $d$ 'autres chercheurs observent une inhibition de la locomotion leucocytaire identique avec des agents intraveineux. Alors que l'enflurane, ne produit, selon les observations, soit aucun effet, soit une amplification de la migration leucocytaire, l'halothane à $1,4 \%$, de son côté, provoque une inhibition de la leucomotion. L'inhibition après anesthésie générale de la migration leucocytaire à la période postopératoire rapportée initialement par Stanley et al., ${ }^{17}$ est subséquement confirmée par Moudgil et al. ${ }^{18} \mathrm{~A}$ l'opposé, on trouve les études in vitro de Duncan et Cullen, ${ }^{19}$ et de Nunn et al. ${ }^{20}$ dont les conclusions ne révèlent aucune baisse de la migration chimiotaxique avec le thiopental ou l'halothane à $2 \%$. Pendant ces études, diverses concentrations d'agents anesthésiques sont utilisées en association avec des méthodes de mesure différentes, ce qui peut avoir pour effet de faire varier les résultats concernant la locomotion leucocytaire. Dans le but d'élucider ces contradictions, Moudgil et al. ont étudié les effets de concentrations équipotentes de plusieurs agents associés au protoxyde d'azote à $70 \%$ sur la migration chimiotaxique des polynucléaires et des monocytes. ${ }^{21}$ Ces recherches révèlent que pour plusieurs agents volatils, le degré d'inhibition à $1 \mathrm{MAC}$ est en rapport avec la nature de l'anesthésique utilisé. A l'exception du protoxyde d'azote, la sévérité de l'inhibition est parallèle à la solubilité lipidique des agents anesthésiques. Dans du protoxyde d'azote à $70 \%$, l'isoflurane affecte la migration chimiotactique des neutrophiles et des monocytes à un degré moindre que le méthoxyflurane. En plus des volatils et des agents d'induction intraveineux, les opiacés dont la morphine, le fentanyl et la mépéridine jouent un rôle inhibiteur de la migration leucocytaire chimiotactique.

Bien que plusieurs études dont la présente, montrent une inhibition de la migration chimiotactique, leur pertinence clinique est loin d'être évidente. Des études antérieures indiquent que la dépression induite par l'anesthésie est de courte durée et facilement renversée. La diminuition des valeurs mesurées sur des spécimens sanguins prélevés en périphérique ne signifie pas nécessairement une inhibition généralisée des défenses de l'hôte. Les résultats peuvent exprimer soit des valeurs altérées, des réserves diminuées ou une immunosuppression clinique franche. Comme la plupart des fonctions immunitaires possèdent des réserves, la dimunution d'une valeur mesurée par un analyse peut n'avoir aucun impact sur la compétence immunitaire totale. Alors qu'Erskine et al. montrent une différence de réaction chimiotactique entre un groupe sous rachianesthésie et l'anesthésie générale et ont spéculé sur un mécanisme pouvant expliquer cette disparité, l'impact global de la diminution de l'immunocompétence sur une population en bonne santé pourrait être négligeable. Du reste, ces auteurs prennent bien soin de ne pas faire de recommandations cliniques basées sur leurs constatations. Les effets provoqués par l'anesthésie sur les composantes spécifiques et non spécifiques de l'immunité pourraient cependant s'appliquer à des populations pédiatriques et gériatriques ou à des traumatisés dont la capacité immunosuppressive est déjà lésée. Afin de définir la nature et le mécanisme de l'immunosuppression provoquée par l'anesthésie et ses conséquences cliniques à la période postopératoire, des recherches additionnelles s'imposent. L'acquisition nouvelles connaissances dans ce domaine pourra un jour contribuer à réduire la morbidité et la mortalité périopératoires d'origine infectieuse.

\section{References}

1 Bernard C. Anesthésiques et l'Asphyxie. Paris: Ballière, 1875.

2 Moudgil GC, Wade AG. Anaesthesia and immunocompetence. Br J Anaesth 1976; 48: 31-9.

3 Duncan $P G$, Cullen BF. Anesthesiology and immunology. Anesthesiology 1976; 45: 522-38.

4 Walton $B$. Anaesthesia, surgery and immunology. Anaesthesia 1978; 33: 322-48.

5 Stevenson GW, Hall SC, Rudnick S, Seleny FL, Stevenson $H S$. The effect of anesthetic agents on the human immune response. Anesthesiology 1990; 72: 542-52.

6 Salo $M$. Effects of anaesthesia and surgery on the immune response. Acta Anaesthesiol Scand 1992; 36: 201-20.

7 Metchnikoff E. Lectures on the Comparative Pathology of Inflammation. London: Kegan Paul, 1893.

8 Boyden SV. The chemotactic effect of mixtures of antibody and antigen on polymorphonuclear leucocytes. J Exp Med 1962; 115: 453-66.

9 Erskine R, Janicki PK, Ellis P, James MFM. Neutrophils from patients undergoing hip surgery exhibit enhanced movement under spinal anaesthesia compared with general anaesthesia. Can J Anaesth 1992; 39: 905-10.

$10 M c$ Cutcheon $M$. Chemotaxis in leucocytes. Physiol Rev 1946; 26: 319-36.

11 Tranquillo RT, Zigmond SH, Lauffenburger DA. Measurement of the chemotaxis coefficient for human neutrophils 
in the under-agarose migration assay. Cell Motil Cytoskeleton 1988; 11: 1-15.

12 Zigmond SH, Hirsch JG. Leukocyte locomotion and chemotaxis. New methods for evaluation and demonstration of cell-derived chemotactic factor. J Exp Med 1973; 137: $387-410$.

13 Islam $L N$, McKay IC, Wilkinson PC. The use of collagen or fibrin gels for the assay of human neturophil chemotaxis. J Immunol Methods 1985; 85: 137-51.

14 Addison IE, Babbage JW. A raft technique for chemotaxis: a versatile method suitable for clinical studies. $\mathbf{J}$ Immunol Methods 1976; 10: 385-8.

15 Nunn JF, Sharp JA, Kimball KL. Reversible effects of an inhalational anaesthetic of lymphocyte motility. Nature (Lond) $1970 ; 226: 85-6$.

16 Moudgil GC, Allan RB, Russell RJ, Wilkinson PC. Inhibition, by anaesthetic agents, of human leucocyte locomotion towards chemical attractants. Br J Anaesth 1977; 49: 97-105.

17 Stanley TH, Hill GE, Portas MR, Hogan NA, Hill HR. Neutrophil chemotaxis during and after general anesthesia and operation. Anesth Analg 1976; 55: 668-73.

18 Moudgil GC, Pandya AR, Ludlow DJ. Influence of anaesthesia and surgery on neutrophil chemotaxis. Can Anaesth Soc J 1981; 28: 232-8.

19 Duncan PG, Cullen BF. Neutrophil chemotaxis and anaesthesia. Br J Anaesth 1977; 49: 345-9.

20 Nunn JF, Sturrock JE, Jones AJ, et al. Halothane does not inhibit human neutrophil function in vitro. $\mathrm{Br} J$ Anaesth 1979; 51: 1101-8.

21 Moudgil GC, Gordon J, Forrest JB. Comparative effects of volatile anaesthetic agents and nitrous oxide on human leucocyte chemotaxis in vitro. Can Anaesth Soc J 1984; 31: 631-7. 